This is the accepted manuscript version of the following article:

A. Agosti, E. Kuna, G. Bergamini, "Divergent terpyridine-based coordination for the construction of photoactive supramolecular structures", Eu. J. Inorg. Chem., 2019, 577-584.

Which has been published in final form at:

https://onlinelibrary.wiley.com/doi/full/10.1002/ejic.201801263

This article may be used for non-commercial purposes in accordance with Wiley Terms and Conditions for Use of Self-Archived Versions.

(C) 2019 Wiley-VCH Verlag GmbH \& Co. KGaA, Weinheim 


\title{
Divergent Terpyridine-Based Coordination for the Construction of Photoactive Supramolecular Structures
}

\author{
Amedeo Agosti, ${ }^{[\mathrm{a}]}$ Ewelina Kuna, ${ }^{[\mathrm{a}]}$ and Giacomo Bergamini*[a]
}

\section{radiation and investigate the interaction of light and matter is} one of the most compelling prospects of our century. Supramolecular chemistry has unraveled the opportunity to synergistically combine the chemical and optoelectronic properties of the most diverse classes of compounds. Among these, ter11 pyridines have acted as pivotal ligand units that enable selfassembly of multicomponent chromophoric systems. In this review we therefore elucidate the metal-coordinating ability of these materials, that promote a plethora of aggregation-induced phenomena. In particular, fluorescence tuning, reversible stimuli-responsive phosphorescence enhancement and lowdimensional complexation have been demonstrated for carbon and sulfur based terpyridine oligomeric structures. Common thread of such processes is the versatile application of light as a trigger for spectroscopic investigation and output for superior optical, medical and sensing devices.

\section{6}

\section{as}

\section{Introduction}

The first publication concerning the chemistry of terpyridine compounds appeared at the beginning of the 1930s, when Morgan and Brustall described the synthesis of 2,6-bis(2-pyr-

26 idyl)pyridine (Tpy). ${ }^{[1,2]}$ It was then demonstrated how this tridentate ligand could create highly stable complexes by chelating a wide range of transition metal ions in their low oxidation states, due to strong metal-ligand $\left(d-\pi^{*}\right)$ back donation. ${ }^{[3]}$ From that moment on, terpyridine has been considered as a suitable

31 building block for supramolecular construction, being extremely versatile in terms of functionalization at every ring-position through various substitutions or interconversion reactions. ${ }^{[4]}$ Over time, terpyridines and their functionalized analogues have been gaining ground on the field of supramolec-

36 ular and coordination chemistry, as well as material science, mainly due to their ability to create stable complexes with appealing optical, electronic, and magnetic properties. ${ }^{[5-8]}$

Interestingly, terpyridine metal complexes $\left[\mathrm{M}(\mathrm{Tpy})_{2}{ }^{\mathrm{n}+}\right]$ usually possess a distorted octahedral coordination geometry,

41 caused by hexacoordinate metal ions, ${ }^{\left[{ }^{[9]}\right.}$ where a linear bilateral conformation of ligands can be obtained by complexation of terpyridine ligands to a metal center in 2:1 ratio. ${ }^{[10]}$ The directionality of these non-covalent bonds is crucial in the design and construction of supramolecular systems, either presenting

46 flexible and rigid polymer-like conformation or geometrically well-defined architectures.

The formation of tailored self-assemblies can be provided by a suitable terpyridine construction strategy, where the coordi-

\footnotetext{
[a] Department of Chemistry "Giacomo Ciamician", University of Bologna, Via Selmi 2, 40126, Bologna, Italy E-mail: giacomo.bergamini@unibo.it https://site.unibo.it/photoactive-system/en/people

(iD $\operatorname{ORCID(s)~from~the~author(s)~for~this~article~is/are~available~on~the~WWW~}$ under https://doi.org/10.1002/ejic.201801263.
}

nation process and structural configuration of the metal-ligand complexes might be affected by the rigidity of the self-assem- 51 bled system. ${ }^{[11]}$ Terpyridines moieties can be linked via a rigid spacer, forming a predictable structure, or by flexible units, yielding a random architecture. ${ }^{[12,13]}$ The possibility to combine various bridging ligands drives different physics and geometry of self-assembling multinuclear complexes, offering diverse 56 supramolecular architectures including grids and racks, ${ }^{[14]}$ macrocycles ${ }^{[15]}$ or metal coordination polymers ${ }^{[16]}$ (Figure 1).

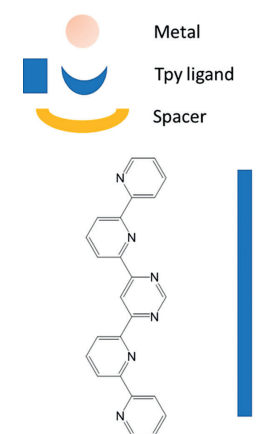

(a)

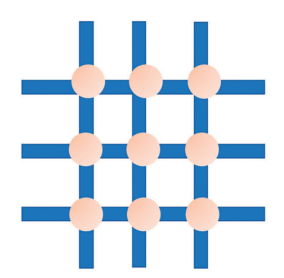

(b)

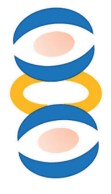

(c)

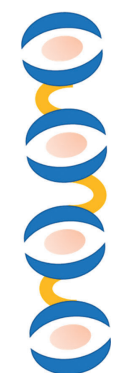

(d)
Figure 1. Schematic representation of a mononuclear (a) racks, (b) grids, (c) macrocycles, and (d) linear metallopolymers.

Likewise, the ligand-metal-ligand connectivity allows to control bond strength and, ultimately, design the supramolecular self-assemblies architecture. ${ }^{[13]}$ Being the formation of terpyr- 61 idine-based structures driven by the interplay between kinetics and thermodynamics driving forces, Tpy ligands can coordinate both labile $\left(\mathrm{Cd}^{2+} ; \mathrm{Zn}^{2+}\right)$ and non-labile $\left(\mathrm{Fe}^{2+} ; \mathrm{Os}^{2+} ; \mathrm{Ru}^{2+}\right)$ metals, with increasing complexation kinetic rates in the order $\left(\mathrm{Cd}^{2+}<\mathrm{Zn}^{2+}<\mathrm{Fe}^{2+}<\mathrm{Os}^{2+}<\mathrm{Ru}^{2+}\right) .{ }^{[17]}$

As a consequence of the high binding affinity, custom-made terpyridine monomers combined with suitable metals give rise 
to programmable and highly ordered $2 \mathrm{D}^{[18-20]}$ and 3D structures. $^{[21-23]}$ These supramolecular materials based on terpyr-

71 idine-containing $\mathrm{p}$-conjugated polymers found application in optoelectronic devices like organic light-emitting diode, ${ }^{[24]}$ photovoltaic cells, ${ }^{[25]}$ organic field-effect transistors ${ }^{[26]}$ and ion sensors. ${ }^{[27,28]}$ Beyond this, the design of reversible and flexible metallo-polymeric systems allows the integration of such archi-

76 tectures even in biological environments (e.g. as biological marker). ${ }^{[29]}$

Another key feature of terpyridine-based materials is related to their photophysical properties. Pristine Tpy shows an absorption spectrum below $300 \mathrm{~nm}$ and weak emission $\left(\Phi_{\mathrm{em}}=0.003\right)$

81 around $330 \mathrm{~nm}$. The successive protonation of the pyridinic nitrogen provides significant changes in both the absorption and emission spectra, boosting the fluorescence quantum yield above $70 \%\left(\Phi_{\text {emTpyH }+}=0.17\right.$ and $\left.\Phi_{\text {emTpy2H2+ }}=0.71\right) .^{[30]}$ In the same way, metal ion complexation causes strong redshifts in

86 the absorption spectra, along with changes in the emission properties, depending on the metal ion involved. Moreover, chemical functionalization of Tpy allows fine tuning of the optical and electronic properties of the ligand and metal complexes. For instance, in order to improve the optical properties

91 and move the absorption and emission spectra to the visible region, the conjugation system of the terpyridine moieties can be extended by attaching appropriate substituents, especially in the $4^{\prime}$ position of terpyridine units. ${ }^{[9]}$ The use of $4^{\prime}$-substituted ligands allows to obtain a linear vector between the two

96 para substitution groups (Figure 2), providing higher control on the energy and electron transfer through ligand-ligand, ligandmetal or metal-ligand interactions. ${ }^{[31]}$

An explanatory example is the case of the $\mathrm{Ru}(\mathrm{Tpy})_{2}{ }^{2+}$. In contrast to bipyridine complexes, ruthenium terpyridine complexes

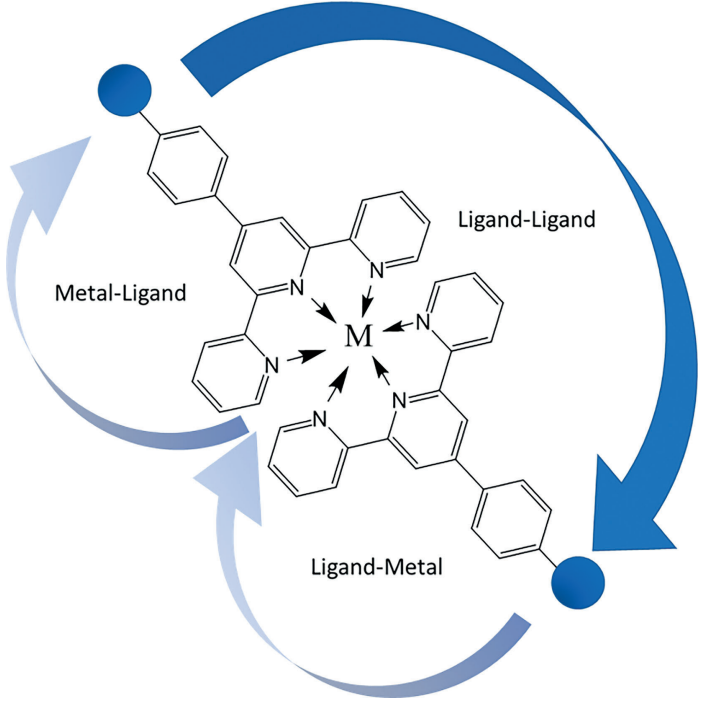

Figure 2. The potential pathway of the energy and electron transfer in 4'substituted terpyridine dyads complex.

do not emit at room temperature due to a non-radiative transi- 101 tion of the excited triplet metal to ligand charge-transfer state $(3 \mathrm{MLCT})$ via a triplet metal centered state (3MC) to the ground state. ${ }^{[32]}$ However, through incorporation of an electron withdrawing or donating group, the energy band gap between triplet states can be widened, improving the photophysical behav- 106 ior. ${ }^{[6]}$ Hence, the various functionalized terpyridine ligands allow to fine-tune the optical properties of the parent mononuclear complexes (dyads or triads) and extended supramolecular frameworks (i.e. polynuclear complexes). In order to enhance the photophysical properties, a variety of chromophores have 111 been appended to the Tpy moiety, thus combining lumines-
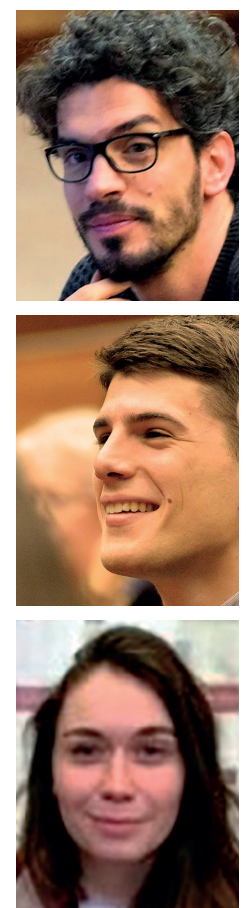

Giacomo Bergamini obtained his PhD in Chemical Science at the University of Bologna in 2007 under the supervision of Prof. Vincenzo Balzani. He currently works in the Photochemical Nanoscience Laboratory in Bologna and is co-author of more than 90 scientific papers in refereed international journals. He is the Coordinator of the H2020-MSCA-ITN-2016 PHOTOTRAIN

Amedeo Agosti studied Materials Science at ETH Zürich, where he obtained his M.Sc degree in 2016. He joined the group of Prof. H.J. Snaith at the University of Oxford for his master thesis, where he worked on perovskite solar cells. Previously, he obtained his B.Sc. degree in Materials and Nanotechnologies Engineering at Politecnico di Milano. Amedeo is currently a PhD student at the University of Bologna under the supervision of Prof. Giacomo Bergamini.

Ewelina Kuna graduated in 2013 from Warsaw University of Technology in Chemical Technology. From 2014 to 2016 she was a research assistant at the Institute of Physical Chemistry Polish Academy of Science in Poland. Ewelina currently is a PhD student at the University of Bologna under the supervision of Prof. Giacomo Bergamini. 
cence and photoinduced energy and/or electron transfer processes with metal binding properties. ${ }^{[5,31]}$

Finally, the peculiar photophysical properties and responsiv116 ity to external stimuli make terpyridines a perfect tool to monitor self-assembly, metal coordination and photoinduced processes. Experimental investigation of absorption changes, emission or lifetime quenching and sensitized emission, can indeed provide information on the stoichiometric ratio, interaction

121 among the different units inside the supramolecular architecture and, ultimately, on the effect of the environment.

This minireview therefore aims at summarizing a set of experimental contributions from our research group, in which terpyridines have been used as binding sites in the construction

126 of 2D and 3D supramolecular structures with intriguing photophysical properties.

\section{C-Based Multiterpyridine Systems}

The most common method to obtain highly luminescent terpyridine molecules is extending the rigidity and $\pi$-conjugation

131 of the system by functionalization of the phenyl-ring in the $4^{\prime}$ position of Tpy units (so called Kröhnke-type terpyridine). ${ }^{[6]}$ Although the nonplanarity between phenyl and terpyridyl rings reduces electron delocalization, $4^{\prime}-p$-tolyl-2,2': $6^{\prime}, 2^{\prime \prime}$-terpyridine (hereafter called TolTpy) exhibits improved luminescence fea-

136 tures in the UV spectral region with $8 \%$ emission quantum yield in organic solvents. Hence, in this section, we report the divergent functionalization of a $\mathrm{sp}^{3}$ carbon and a benzene with multiple TolTpy units, which, upon addition of specific metal ions, self-assemble in nanostructures.

\section{2.1 Tetrahedral Shaped}

A first example of C-based multiterpyridines is represented by a tetrahedral core functionalized with four Tpy units (TTT; Figure 3).

The $\mathrm{sp}^{3}$ core conveys a tetrahedral symmetry to the rigid 146 molecule, spatially segregating the Tpy moieties and thus pre-

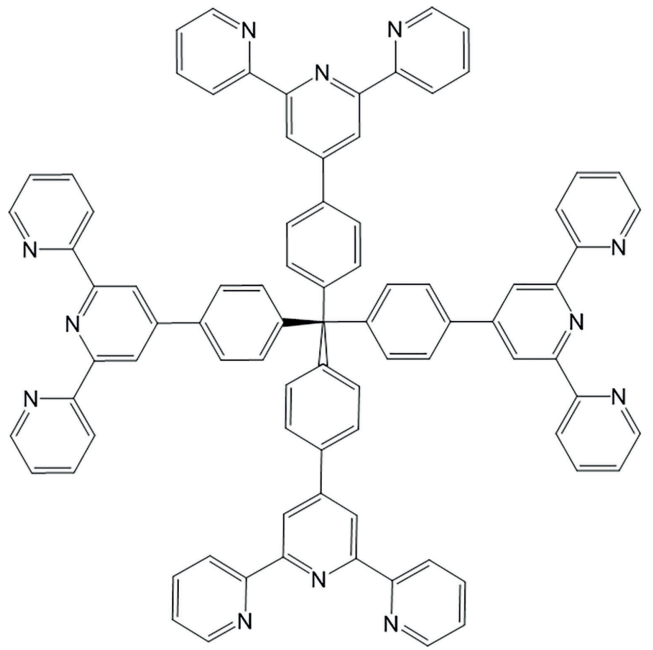

venting intramolecular 2:1 Tpy: $\mathrm{Mn}^{+}$complexation. Exploiting the strong chelating activity of the Tpy binding pocket, the complexation of TTT with $\mathrm{Fe}^{2+}$ and $\mathrm{Ln}^{3+}$ ions was investigated with the aim of determining the stoichiometry, stability and photophysical properties of the formed species. The addition of 151 $\mathrm{Fe}\left(\mathrm{CF}_{3} \mathrm{SO}_{3}\right)_{2}$ to a diluted solution of TTT in dichloromethane generates significant changes in the absorption spectra, namely a bathochromic shift of the Tpy absorption band at $300 \mathrm{~nm}$, characterized by an isosbestic point at $328 \mathrm{~nm}$, associated with the appearance of a MLCT band at $575 \mathrm{~nm}$. The corresponding 156 emission spectra, obtained through excitation at the isosbestic point, showed strong quenching of the fluorescence band at $380 \mathrm{~nm}$. Analysis of the UV/Vis traces variation shows a titration plateau when 2.0 eqs of $\mathrm{Fe}^{2+}$ with respect to TTT were added to the solution, or at half eq. of $\mathrm{Fe}^{2+}$ for each terpyridine unit. 161 Consequently, the metal to ligand charge transfer (MLCT) band for Fe-TTT complex showed 4-fold increase in the molar absorptivity, compared to the simple $\left[\mathrm{Fe}(\mathrm{Tpy})_{2}\right]^{2+}$ complex. ${ }^{[33]}$ From these results, we can assume that all the terpyridine units present in TTT molecules are engaged in metal complexation 166 with 2:1 Fe/TTT stoichiometry. Moreover, it was noticed that the emission intensity, detected via excitation at the isosbestic point, is not superimposed on that of the absorption decrease at $300 \mathrm{~nm}$. Following the addition of 1 eq. of $\mathrm{Fe}^{2+}$, absorption settles indeed to $50 \%$, while the emission intensity of TTT li- 171 gand decreases to $10 \%$, likely suggesting an interaction, occurring in the excited state, between free and complexed Tpy units. This hypothesis was corroborated by nanosecond lifetime investigation of the excited state, together with the use of low concentration of TTT, which ruled out the intervention of inter- 176 molecular interactions. Furthermore, taking into consideration the stoichiometry and the fact that no precipitation was observed, we speculated the formation of nanometer-sized assemblies. The resulting oligomeric structure, probed by Dynamic Light Scattering (DLS) and Atomic Force Microscopy (AFM), con- 181 sists indeed of particles of approximately $20 \mathrm{~nm}$ diameter. Finally, aggregation of these nanoparticles gradually leads to formation of "polymer" chains (up to $5 \mu \mathrm{m}$ long) that are stable

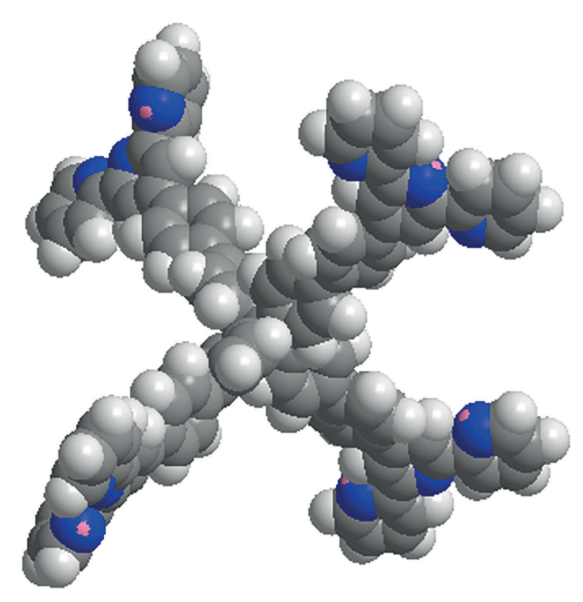

Figure 3. 2D and 3D structure of TTT ligands. ${ }^{[33]}$ 

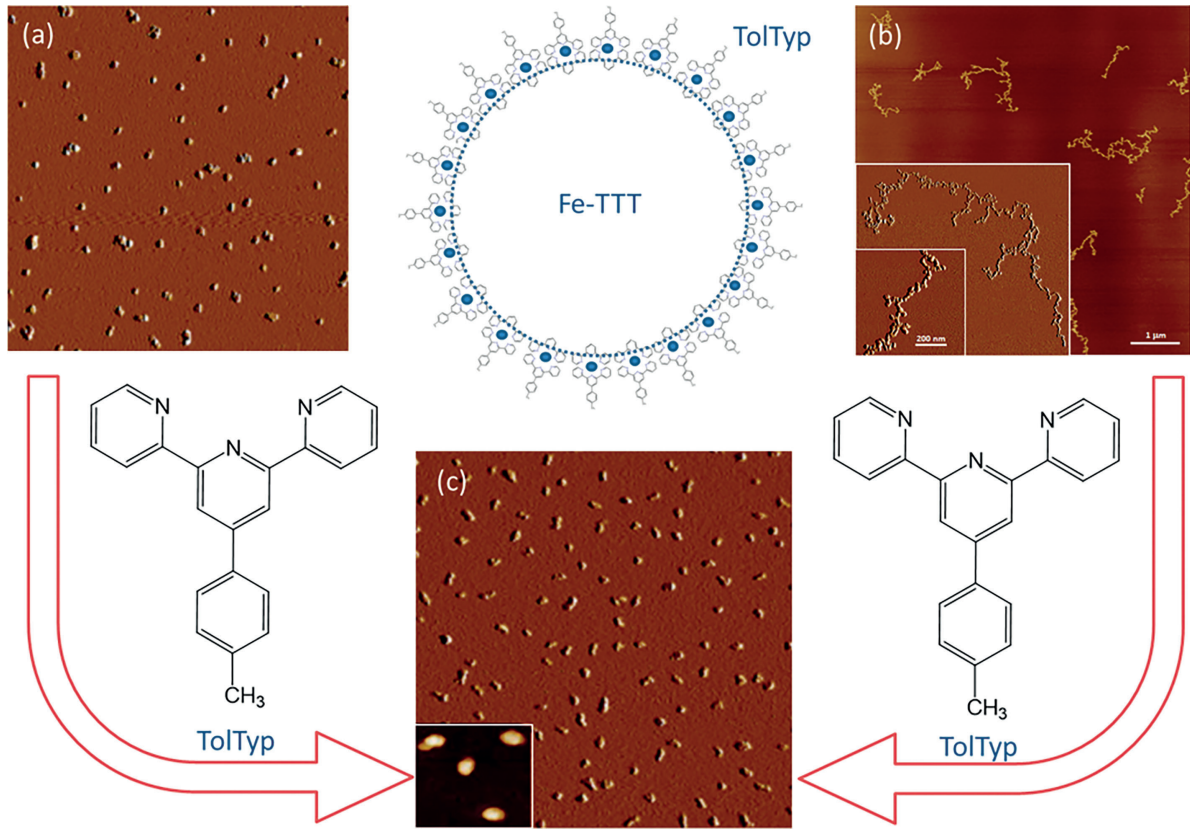

Figure 4. AFM images of (a) Fe-TTT particles; (b) polymeric chain of aggregates; (c) individual particle after the addition capping agent (TolTpy).

for weeks without further precipitation. The "polymerization" 186 process is triggered by the presence of the free metal coordination sites located on the nanoparticles surface, providing quasifractal structures peculiar to diffusion-controlled aggregations. ${ }^{[33]}$

Interestingly, aggregation proceeds along discrete axes,

191 maintaining the original particles within the assembly, and does not occur via formation of globular particulate entities. Further analyses are required to provide a comprehensive description of the process mechanism, however it is safe to propose that aggregation does not proceed randomly, nor depends on con-

196 centration. Remarkably, polymerization may eventually be halted by the addition of TolTyp units as a capping agent, allowing self-assembly control from molecular up to mesoscopic scale (Figure 4).

Subsequently, the investigation of TTT molecule optical and 201 chemical properties addressed the self-assembly behavior in relation to luminescence properties of TTT- $\mathrm{Mn}^{+}\left(\mathrm{Mn}^{+}=\mathrm{Zn}^{2+}\right.$, $\left.\mathrm{Eu}^{3+}, \mathrm{Nd}^{3+}, \mathrm{Yb}^{3+}\right){ }^{[34]}$ First of all, we confirmed the formation of 2:1 $\mathrm{Mn}^{+}$:TTT complexes, monitoring the changes in the absorption and emission spectra, as well as stable assemblies around

$20650 \mathrm{~nm}$ in size (by DLS and AFM measurements). Of particularly interest is the emission intensity evolution, characterized by the complete quenching of the TTT emission and concomitant increase in the $\mathrm{Zn}^{2+}, \mathrm{Eu}^{3+}, \mathrm{Nd}^{3+}$ and $\mathrm{Yb}^{3+}$ complexes emission, spanning from the visible to the near infrared (NIR) region (Fig211 ure 5).

The addition of TolTpy as capping agent to the particles, not only improved the stability and prevented further polymerization, but also improved the emission properties of the Eu ${ }^{3+}$-TTT and $\mathrm{Nd}^{3+}$-TTT nanostructures. The complexation of two differ-

216 ent luminescent metal ions by TTT ligands results in the formation of dual emissive particles. Notably, the addition of 0.2 equivalents $\left[\mathrm{Nd}\left(\mathrm{CF}_{3} \mathrm{SO}_{3}\right)_{3}\right]$ to a solution of TTT.2Eu $u^{\text {III }}$ decreased

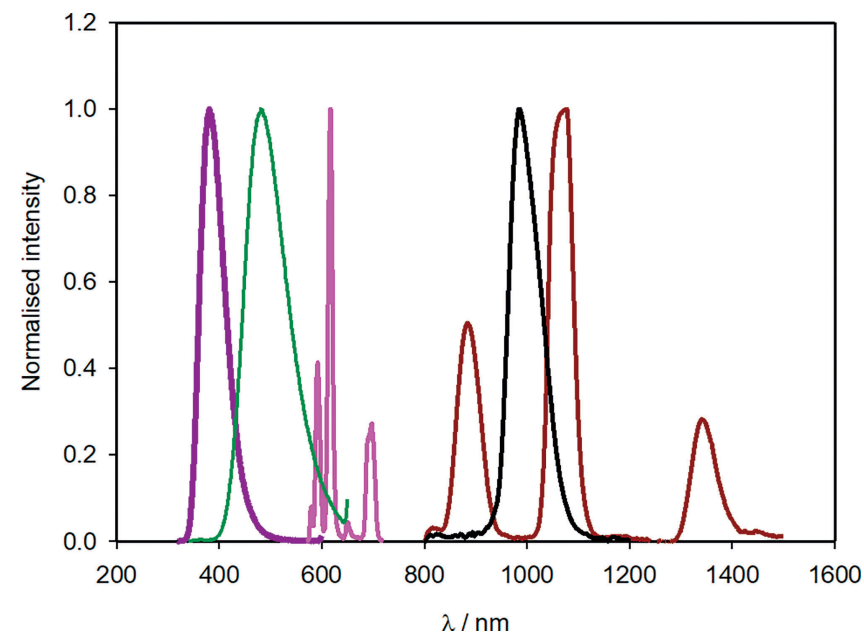

Figure 5. Normalized emission spectra of TTT (purple line), TTT·2Znll (green line), TTT.2Eulll (violet line), TTT.2NdIII (brown line) and TTT.2Yblll (black line) in $\mathrm{CH}_{2} \mathrm{Cl}_{2} \cdot{ }^{[34]}$

the $\mathrm{Eu}^{3+}$ emission by $64 \%$ and the associated emission of the $\mathrm{Nd}^{3+}$ ion in the NIR region. Analysis of the quenching and sensitization rates confirmed that such process is not a mere dis- 221 placement of $\mathrm{Eu}^{3+}$ ions by $\mathrm{Nd}^{3+}$ ions, otherwise a decrease of $\mathrm{Eu}^{3+}$ phosphorescence of $20 \%$ should have been observed. DLS and AFM measurements confirmed the presence of $50 \mathrm{~nm}$ particles and therefore the unchanged size or arrangement of the oligomeric structures. Hence, the possibility of combining dif- 226 ferent metal centers allows to design heterometallic multinuclear complexes with multiple emission.

\subsection{Planar Shaped}

The functionality of TolTpy unit was also investigated when attached to a benzene core, resulting in a rigid and planar struc- 231 


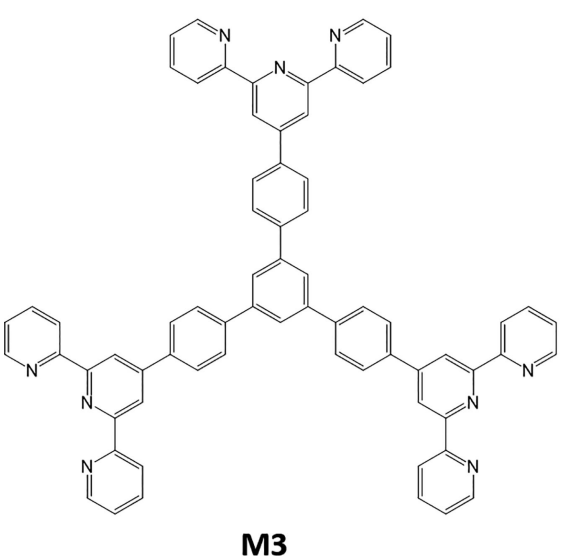

M3

Figure 6. Chemical structure of M3 and M6 multiterpyridine ligands.

ture with superior luminescence properties $\left(\Phi_{\mathrm{em}} \approx 35 \%\right.$ ), containing 3 and 6 binding sites (hereafter called M3 and M6) (Figure 6). To this extent, M6 molecules were previously employed in the construction, at air-liquid interfaces, of free-standing 236 monolayer sheets grasped together by metal ions. ${ }^{[35,36]}$

In particular, our research group contributed to the study of the behavior of M3 and M6 as multiterpyridine ligands for $\mathrm{Zn}^{2+}$ and $\mathrm{Fe}^{2+}$ ions in dichloromethane solution. At first, we demonstrated the formation of 2:1 Tpy: $\mathrm{M}^{2+}$ complexes, for both metal

241 ions and in a low concentration regime, which eventually produced a polymeric structure characterized by multiple ligands organized in coordinative bonds. Later, a detailed analysis of the proposed polymer-like compound and its spatial arrangement was performed by modelling of the speculated structure.

246 As previously described, monitoring of the optical absorption features allowed the observation, for $\mathrm{M}^{2+}$ and $\mathrm{M6}$, of single metal ion bonded to two Tpy units belonging to distinct ligands. In this case, the plateau was reached upon the addition of 3 eqs of $\mathrm{M}^{2+}$ per $\mathrm{M} 6$ unit, thus at half eq. of metal ions per

251 Tpy moiety. Finally, by integrating the photophysical results with computational modelling of the $2 \mathrm{D}$ and $3 \mathrm{D}$ structures, it was possible to infer that, with reference to previous studies conducted at the air-water interface, a predominant 2D grid is formed in solution (Figure 7), nonetheless the final structure 256 probably resulted in a folded configuration. Remarkably, our
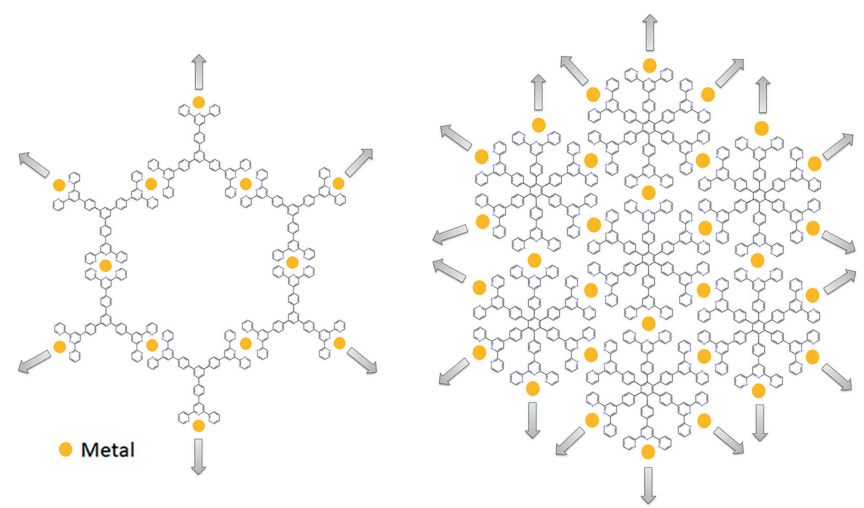

Figure 7. Schematic representation of a $2 \mathrm{D}$ structure formed by $M 3$ and $M 6$.

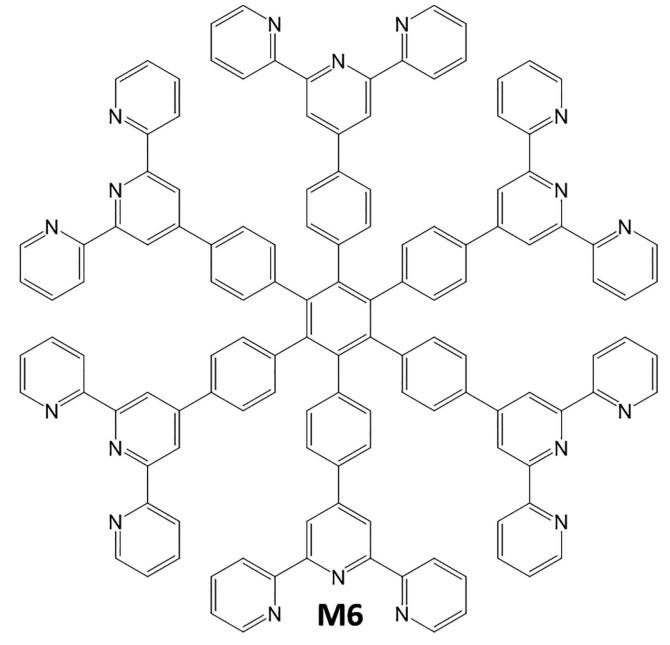

findings reveal that the concentration of 3D deffects, despite being present, was evaluated to be rather negligible compared to the overall metal to ligand ratio.

Overall, these results shed new light on the formation of 2D metal-coordinated polymers and will hopefully trigger a body 261 of research in the application of such materials in the field of molecular sieves, ultrasensitive pressure-sensors, and surface coatings. $^{[8]}$

\section{ArS-Based Multiterpyridine Systems}

In this section we report the description of two examples of 266 multiterpyridine systems based on two particular organic luminophores: hexathiobenzene ${ }^{[37]}$ and tetrathiopyrene, ${ }^{[38]}$ which are attractive candidates for applications like organic light-emitting diodes, field-effect transistors, liquid crystals, and many other solid-state devices.

\subsection{Hexathiobenzene}

Firstly, we synthesized a multichromophoric supramolecular system characterized by six phenylterpyridine units attached through a sulfur atom to a benzene core (BzSTpy, Figure 8). The core of our molecule, more specifically a hexathiobenzene 276 molecule decorated with six tolyl groups, exhibits outstanding phosphorescence properties both in the solid state $\left(\Phi_{\mathrm{em}}\right.$ ca. $100 \%$ and $\tau=4 \mathrm{~ms})^{[37]}$ and when dispersed in a matrix at 77$110 \mathrm{~K}$, but does not reveal any luminescence in liquid solution at room temperature. The emission "onset" was justified accord- 281 ing to intramolecular rotations restriction, a behavior also known as Aggregation Induced Emission (AIE). This atypical emission can be observed either in the solid state or in extremely diluted matrices at sufficiently low temperature (77$110 \mathrm{~K}$ ), both scenarios being characterized by a rigid cage-like 286 environment with limited intramolecular rotations. Therefore, we proposed the integration of terpyridine ligands in a multicomponent chromophore (BzSTpy Figure 8) with the aim of boosting the luminescence properties of the aforementioned compound. Upon addiction of appropriate divalent metal ions 291 


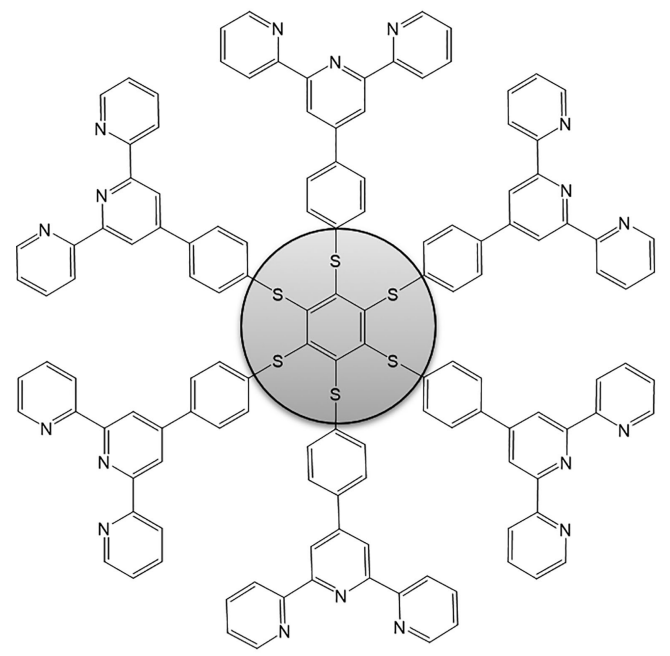

BzSTpy

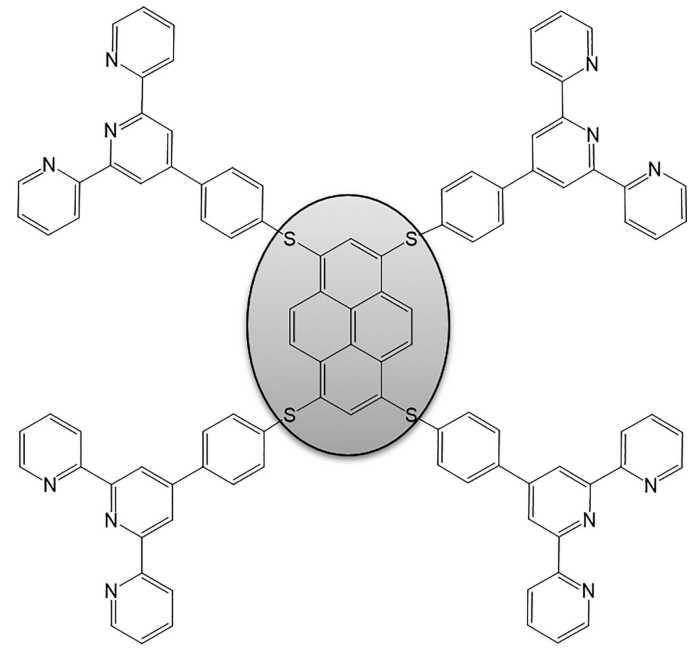

PySTpy

Figure 8. Chemical structure of BzSTpy and PySTpy.

to a solution of BzSTpy, a supramolecular polymer with a 3:1 $\mathrm{Mn}^{+} / \mathrm{X}$ stoichiometry is formed. Particularly interesting is the case of $\mathrm{Mg}^{2+}$ ions, which self-assemble into a bright $\left(\Phi_{\mathrm{em}}=\right.$ $10 \%)$ phosphorescent supramolecular polymer, with microsec296 ond-excited state lifetime in air-equilibrated THF solution. Remarkably, the spectral trace of the compound in solution perfectly matches that of the powder, corroborating the formation of an aggregated coordination compound with reduced intramolecular rotations and motions, thus enhanced phosphores301 cence. In addition to that, upon excitation of the $\left[\mathrm{Mg}(\mathrm{Tpy})_{2}\right]^{2+}$ units of the polymeric structure, sensitization of the core phosphorescence takes place with $>90 \%$ efficiency. Moreover, by addition of $\mathrm{Fe}^{2+}, \mathrm{Co}^{2+}, \mathrm{Ni}^{2+}$, and $\mathrm{Cu}^{2+}$ no phosphorescence was detected, because of the expected photoinduced energy/elec306 tron transfer processes between the phosphorescent core and
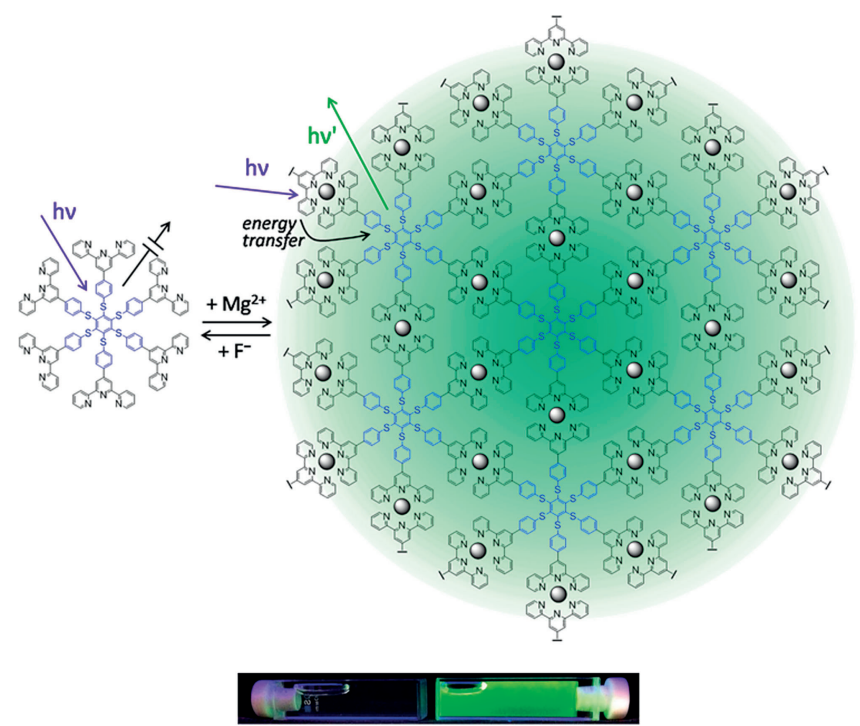

Figure 9. Schematic representation of the supramolecular polymer and the energy transfer process involved. Bottom: pictures of the monomeric and polymeric solutions. the low energy excited states of the metal complexes at the periphery. Nevertheless, these phenomena are not favored in presence of $\mathrm{Ca}^{2+}, \mathrm{Zn}^{2+}$, and $\mathrm{Cd}^{2+}$, which are $\mathrm{d}^{0}$ or $\mathrm{d}^{10}$ metal ions, yielding instead a phosphorescence band observed for $\mathrm{Mg}^{2+}$ with significantly reduced efficiency. Ultimately, we also 311 reported the reversible complexation behavior of the BzSTpy $\cdot \mathrm{Mg}^{2+}$ structure when tetrabutylammonium fluoride was added to the liquid solution. Altogether, the BzSTpy compound benefits from ion complexation not only in the formation of a luminescent supramolecular polymer, but also from the reversi- 316 ble assembly of such structure, opening its utilization as a lightresponsive sensor for both anion and cation (Figure 9).

\subsection{Tetrathiopyrene}

Another example of ArS-based multiterpyridine concerned the investigation of the compound composed by four terpyridine 321 units connected to a tetra(phenylthio)pyrene core (PySTpy, Figure 8). The superior photophysical and electrochemical properties of 1,3,6,8-tetrasubstituted pyrene core, together with its specific geometry, indeed contributed to its wide use in several technological fields ${ }^{[39]}$ and resulted to be highly desirable also 326 for our investigation purposes. The optical features of tetra( $p$-tolylthio)pyrene can be easily identified in the absorption spectrum between 380 and $470 \mathrm{~nm},{ }^{[40]}$ while TolTpy produces the band at $295 \mathrm{~nm}$. These distinct spectral signatures evidence the lack of significant pyrene/terpyridine ground-state interac- 331 tion; as a result of this PySTpy can be safely considered a supramolecular specie. On the other hand, the emission spectra, distinctly obtained by selective excitation of Tpy (UV region) and Pyrene (visible region) moieties, are identical and show an intense florescence band with a maximum at $460 \mathrm{~nm}\left(\Phi_{\mathrm{em}}=336\right.$ $51 \%$ and $\tau=1.2 \mathrm{~ns}$ ) attributable at the tetra(p-tolylthio)pyrene core. Owing to the fact that no TolTpy emission was detected and considering the perfect overlap between excitation $\left(\lambda_{\mathrm{em}}=\right.$ $460 \mathrm{~nm}$ ) and absorption spectrum of PySTpy, it was inferred 
341 that intramolecular energy transfer not only occurs with unitary efficiency, but also with specific directionality, namely from peripheral chromophores to the core.

Next, metal ion complexes formation was observed via addition of $\mathrm{Fe}^{2+}, \mathrm{Zn}^{2+}$, and $\mathrm{Nd}^{3+}$ salts in diluted solutions. In particu-

346 lar, an intermolecular 2:1 Tpy: $\mathrm{M}^{\mathrm{n}+}$ complex organizes, thanks to the divergent geometry and specific stoichiometry, in a "super" oligomeric structures built on $\left[\mathrm{M}(\mathrm{Tpy})_{2}\right]^{n+}$ interactions. DLS and AFM experiments further defined such stable nanoparticles to possess hydrodynamic diameters between 50 and $80 \mathrm{~nm}$, de-

351 pending on the metal ion employed. Interesting results have been obtained through investigation of the photoinduced processes in the final metal-coordinated supramolecular structure.

As already stated, all the metals employed form nanometric structures and the emission of the core is completely quenched

356 after the addition of 2 eqs of metal ions. This behavior can be ascribed to photoinduced energy and/or electron transfer process from the pyrene excited state and the metal complex formed. In the case of $\mathrm{Fe}^{2+}$, the presence of fast non-radiative decay channel, characteristic of the lowest energy MLCT excited

361 state of the pristine $\left[\mathrm{Fe}(\mathrm{Tpy})_{2}\right]^{2+}$ complex, determines the expected fluorescence intensity quenching. On the other hand, the interaction between $\mathrm{Zn}^{2+}$ and PySTpy is characterized by a concomitant decrease of the band of the core at $460 \mathrm{~nm}$ and rise of a very weak $\mathrm{Zn}$-Tpy emission band at $540 \mathrm{~nm}$. The meas-

366 urements of the excitation spectrum, fixing $\lambda_{\mathrm{em}}=540 \mathrm{~nm}$, perfectly matches the absorption spectrum, confirming the occurrence of directional energy transfer from the outer to the inner region of the Zn-PySTpy system.

Finally, similar results have been obtained in the case of $371 \mathrm{Nd}^{3+}$, where the addition of 2 eqs of the $\mathrm{Ln}$ ion causes complete quenching of the core emission and boosts sensitized emission of the $\mathrm{Nd}^{3+}$ ions in the NIR region. Also in this case, core to metal complexes energy transfer with unitary efficiency has been measured.

376 To sum up, in the present study we demonstrated that the addition of different metals ions not only controls the formation

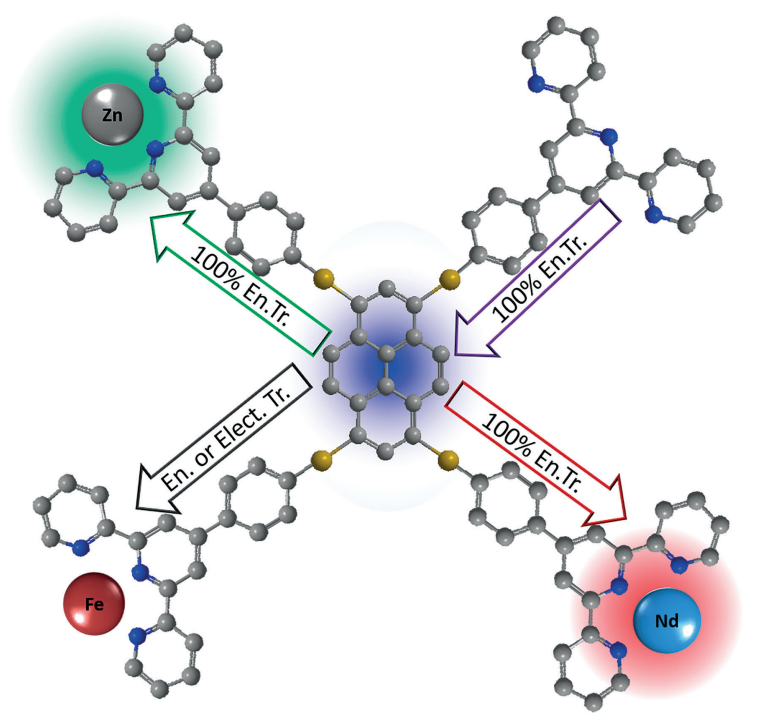

Figure 10. Schematic representation of the intramolecular energy and/or electron transfer processes. of three-dimensional nanoscopic objects, but also switches the direction of the intramolecular energy transfer (Figure 10) in a dual function.

\section{Conclusion}

This review has endeavored to highlight the exceptional versatility of the $2,2^{\prime}: 6^{\prime}, 2^{\prime \prime}$-terpyridine metal-binding domain in coordination chemistry. This class of molecular ligands has been widely investigated owing to their ability to form highly stable complexes following d-block metal ion coordination, featuring 386 unprecedented optoelectronic, magnetic, and electrochemical properties. In particular, we aimed to raise attention on the role of light-matter interaction for this class of compounds. The generation of light by fluorescence or phosphorescence are powerful resources extensively used in the fields of medicine, environ- 391 mental chemistry, sensing, and several others. Furthermore, under a more fundamental point of view, luminescence signals enable extremely sensitive investigation of dendritic structures and superstructures. Therefore, the present review has highlighted the capitalization of light both as a tool for fundamental 396 research and as an output enabling cutting-edge optical technologies.

At first, fluorescence modulation was demonstrated for the family of terpyridines including carbon-based units. Emission from these chromophores can indeed be tuned from the visible 401 to the NIR and, by judicious choice of the metal ions, even simultaneously occurs in dual regions thanks to distinct multinuclear complexation sites. Surprisingly, oligomer formation of such structures retained the morphology of the primitive unit, proceeding along discrete axes and without globular particu- 406 late development. A similar remarkable assembly behavior was described for multi-Tpy ligand systems branching out of a benzene core. Specifically, the supramolecular architectures ensuing from complexation arise in the form of a reticulated monolayer, having metal ions as net points, as neatly confirmed by absorp- 411 tion spectroscopic studies. In addition to that, depending on the nature of the metal used, the structure can undergo transmetalation processes, both in random or directed fashion, still retaining the polymeric integrity.

Finally, our research group benefited from terpyridine com- 416 pounds in the construction of light-harvesting antenna systems and highly luminescent supramolecular polymers. In the former case, a supramolecular, metal coordinated, polymer structure based on a tetrathiophene core revealed $100 \%$ efficient directional energy transfer from the periphery to the central units. 421 This process occurs thanks to $\left[\mathrm{M}(\mathrm{Tpy})_{2}\right]^{n+}$ interactions and was demonstrated for an arsenal of metal ions, namely $\mathrm{Fe}^{2+}, \mathrm{Zn}^{2+}$, $\mathrm{Nd}^{3+}$, enabling broad fluorescence tuning. Additionally, metal ion coordination allowed sensing features when Tpy units were appended to sulfurated benzene core compounds. Through the 426 reversible formation of a polymeric antenna, phosphorescence was indeed turned on and off thanks to the AIE effect.

To conclude, the research just described should highlight the phenomenal adaptability of the terpyridine unit to a host of chromophoric structures, boosting their structural and opto- 431 electronic properties in fluid solution. We also envisage an in- 
crease in the use of light as a powerful tool to critically investigate supramolecular compounds and their application to a broad range of energy and medical applications.

\section{Acknowledgments}

We gratefully acknowledge the H2020-MSCA-ITN-2016 (722591PHOTOTRAIN).

\section{Keywords: Terpyridine · Supramolecular chemistry} Luminescence · Polymers

441 [1] G. T. Morgan, F. H. Burstall, J. Chem. Soc. 1931, 0, 20-30.

[2] S. G. Morgan, F. H. Burstall, J. Chem. Soc. 1937, 0, 1649-1655.

[3] U. S. Schubert, C. Eschbaumer, Angew. Chem. Int. Ed. 2002, 41, 2892 2926; Angew. Chem. 2002, 114, 3016.

[4] U. S. Schubert, A. Winter, G. R. Newkome, $\square((<=\square$ author: please 446 provide the title of this book $\square$ )) $\square$, Chapter 2 (Eds.: U. S. Schubert, A. Winter, G. R. Newkome), Wiley-VCH, Verlag, 2011, pp. 2-10.

[5] U. S. Schubert, H. Hofmeier, G. R. Newkome, Modern Terpyridine Chemistry; Wiley-VCH: Weinheim, Germany, 2006.

[6] A. Wild, A. Winter, F. Schlütter, U. S. Schubert, Chem. Soc. Rev. 2011, 40, 1459-1511.

[7] E. C. Constable, Chem. Soc. Rev. 2007, 36, 246-253.

[8] M. E. Gallina, G. Bergamini, S. Di Motta, J. Sakamoto, F. Negri, P. Ceroni, Photochem. Photobiol. Sci. 2014, 13, 997-1004.

[9] I. M. Dixon, J. P. Collin, J. P. Sauvage, L. Flamigni, S. Encinas, F. Barigelletti, 456 Chem. Soc. Rev. 2000, 29, 385-391.

[10] J. Veliks, J.-C. Tseng, K. I. Arias, F. Weisshar, A. Linden, J. S. Siegel, Chem. Sci. 2014, 5, 4317-4327.

[11] T. Tokizaki, T. Toyoshima, S. Watanabe, Synth. Commun. 2015, 45, 127136.

461 [12] S. S. M. Fernandes, M. Belsley, C. Ciarrocchi, M. Licchelli, M. Manuela, M. Raposo, Dyes Pigm. 2018, 150, 49-58.

[13] S. Chakraborty, W. Hong, K. J. Endres, T. Z. Xie, L. Wojtas, C. N. Moorefield, C. Wesdemiotis, G. R. Newkome, J. Am. Chem. Soc. 2017, 139, 3012-3020.

[14] A. M. Stadler, N. Kyritsakas, R. Graff, J. M. Lehn, Chem. Eur. J. 2006, 12, 466 4503-4522

[15] T. Brandl, V. Hoffmann, A. Pannwitz, D. Häussinger, M. Neuburger, O. Fuhr, S. Bernhard, O. S. Wengera, M. Mayor, Chem. Sci. 2018, 9, 3837-3843.

[16] N. A. Tavenor, M. J. Murnin, W. S. Horne, J. Am. Chem. Soc. 2017, 139, 2212-2215.

471 [17] S. Chakraborty, G. R. Newkome, Chem. Soc. Rev. 2018, 47, 3991-4016.

[18] X. Feng, A. D. Schlüter, Angew. Chem. Int. Ed. 2018, 57, 13748-13763; Angew. Chem. 2018, 130, 000-000.

[19] S. Chakraborty, R. Sarkar, K. Endres, T. Z. Xie, M. Ghosh, C. N. Moorefield, M. J. Saunders, C. Wesdemiotis, G. R. Newkome, Eur. J. Org. Chem. 2016,
[20] G. R. Newkome, P. Wang, C. N. Moorefield, T. J. Cho, P. Mohapatra, S. Li, S.-H. Hwang, O. Lukoyanova, L. Echegoyen, J. A. Palagallo, V. Iancu, S.-W. Hla, Science 2006, 312, 1782-1785.

[21] T. Z. Xie, K. J. Endres, Z. Guo, J. M. Ludlow, C. N. Moorefield, M. J. Saunders, C. Wesdemiotis, G. R. Newkome, J. Am. Chem. Soc. 2016, 138, 481 12344-12347.

[22] Y. C. Wang, Y. P. Liang, J. Y. Cai, Y. J. He, J. Lee, Y. T. Chan, Chem. Commun. 2016, 52, 12622-12625.

[23] C. Zhang, R. S. Patil, C. Liu, C. L. Barnes, J. L. Atwood, J. Am. Chem. Soc. 2017, 139, 3012-3020Ref. [13] $((<=\square$ author: here is a duplicate 486 with ref. [13] $\square)$ ( $\square$.

[24] C. Fan, X. Wang, J. Luo, Opt. Mater. 2017, 64, 489-495.

[25] D. Saccone, C. Magistris, N. Barbero, P. Quagliotto, C. Barolo, G. Viscardi, Materials 2016, 9, 1-37.

[26] J. Kwon, J. P. Hong, S. Noh, T. M. Kim, J. J. Kim, C. Lee, S. Lee, J. I. Hong, 491 New J. Chem. 2012, 36, 1813-1818.

[27] D. Maity, C. Bhaumik, D. Mondal, S. Baitalik, Inorg. Chem. 2013, 52, 13941-13955.

[28] L. Taihong, L. Ke, Z. Jinling, W. Zhaolong, ChemistrySelect 2018, 3, 55595565 .

[29] J. M. Lynam, Dalton Trans. 2008, 0, 4067-4078.

[30] G. Albano, V. Balzani, E. C. Constable, M. Maestri, D. R. Smith, Inorg. Chim. Acta 1998, 277, 225-231

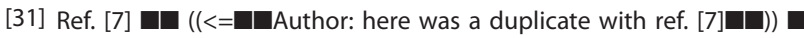

[32] J. P. Sauvage, J. Paul Collin, J. C. Chambron, S. Guillerez, C. Coudret, V. Balzani, F. Barigelletti, L. De Cola, L. Flamigni, Chem. Rev. 1994, 94 993-1019.

[33] J. K. Molloy, P. Ceroni, M. Venturi, T. Bauer, J. Sakamoto, G. Bergamini, Soft Matter 2013, 9, 10754-10758.

[34] J. K. Molloy, Z. Pillai, J. Sakamoto, P. Ceroni, G. Bergamini, Asian J. Org. 506 Chem. 2015, 4, 251-255.

[35] T. Bauer, Z. Zheng, A. Renn, R. Enning, A. Stemmer, J. Sakamoto, A. D. Schlüter, Angew. Chem. Int. Ed. 2011, 50, 7879-7884; Angew. Chem. 2011, 123, 7879-7884.

[36] Z. Zheng, C. S. Ruiz-Vargas, T. Bauer, A. Rossi, P. Payamyar, A. Schütz, A. 511 Stemmer, J. Sakamoto, A. D. Schlüter, Macromol. Rapid Commun. 2013, 34, 1670-1680.

[37] G. Bergamini, A. Fermi, C. Botta, U. Giovanella, S. Di Motta, F. Negri, R. Peresutti, M. Gingras, P. Ceroni, J. Mater. Chem. C 2013, 1, 2717-2724.

[38] M. Gingras, V. Placide, J.-M. Raimundo, G. Bergamini, P. Ceroni, V. Balzani, 516 Chem. Eur. J. 2008, 14, 10357-10363.

[39] S. Yoshimitsu, T. Mutai, I. Yoshikawa, K. Araki, J. Am. Chem. Soc. 2007, 129, 1520-1521; P. Sonar, M. S. Soh, Y. H. Cheng, J. T. Henssler, A. Sellinger, Org. Lett. 2010, 12, 3292-3295.

[40] G. Heywang, F. Jonas, Eur. Pat. Appl., 1989, pat no. EP0339419A3.

Received: October 15, 2018 
Supramolecular Architectures

A. Agosti, E. Kuna,

${ }^{526}$ G. Bergamini* $1-9$

(D) Divergent Terpyridine-Based Coor531 dination for the Construction of Photoactive Supramolecular Structures

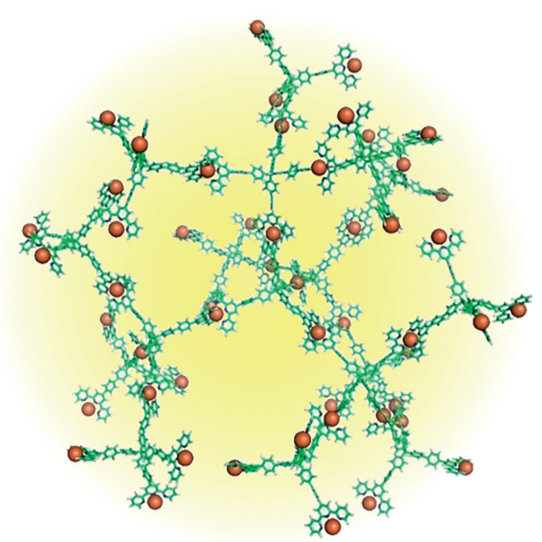

Terpyridine is one of the most intriguing building blocks in the construction of photoactive supramolecular architectures, and light is the perfect tool to investigate their structure and to exploit optical and electronic functions.

536 Authors: Please check that the ORCID identifiers listed below are correct. We encourage all authors to provide an ORCID identifier for each coauthor. ORCID is a registry that provides researchers with a unique digital identifier. Some funding agencies recommend or even require the inclusion of ORCID IDs in all published articles, and authors should consult their funding agency guidelines for details. Registration is easy and free; for further information, see http://orcid.org/.

Amedeo Agosti

541 Ewelina Kuna

Giacomo Bergamini http://orcid.org/0000-0002-2135-4073*

DOI: 10.1002/ejic.201801263 